\title{
Influence of $\beta$-cyclodextrin as an encapsule and as an inclusion complex dopant on conducting polyaniline
}

\author{
E SUBRAMANIAN*, G ANITHA, M KARTHIK SELVAM and \\ M IBRAHIM ALI BADUSHA \\ Department of Chemistry, Manonmaniam Sundaranar University, Abishekapatti, Tirunelveli 627 012, India
}

MS received 19 July 2003; revised 10 May 2004

\begin{abstract}
An investigation on the effect of $\beta$-cyclodextrin (CD) in both free and inclusion-complexed forms with a guest anionic metal complex, dioxalatodiaquochromate(III) (DDC), on the characteristics of conducting polyaniline (PANI) is carried out. Four materials, PANI (i.e. PANI-SO ${ }_{4}^{2-}$ ), PANI-DDC, PANI-CD and PANICD + DDC were prepared by in situ chemical oxidative polymerization in aqueous $\mathrm{H}_{2} \mathrm{SO}_{4}$ at pH 1 and subjected to electrical conductivity and spectral (IR and UV-vis) measurements. DDC and CD when separately incorporated, reduce the conductivity of PANI by about half whilst their inclusion complex CD + DDC enhances it. Spectral characterization reveals that DDC as a dopant and CD as an encapsule exhibit their effects through adverse interaction with imine-amine $N$ centres and benzenoid moiety of PANI. The inclusion complex CD + DDC, on the contrary, functions as a dopant by lying in between the chains and seems to promote the extended conformation of PANI chain and hence the $\pi$-electron delocalization. Exposure of the material to methanol vapour causes a decrease in conductivity in PANI and PANI-CD while an increase in PANICD + DDC. This study makes explicit the distinct role of CD as an encapsule and CD + DDC inclusion complex as a dopant in altering the electrical property of PANI.
\end{abstract}

Keywords. Polyaniline; $\beta$-cyclodextrin; dioxalatodiaquochromate(III); inclusion complex; characteristics development.

\section{Introduction}

Conducting polyaniline has received a great deal of attention worldwide for the past two decades, partly due to its uniqueness arising from its chemical flexibility of imineamine $\mathrm{N}$ centres and the associated protonation dependent conductivity and partly due to its environmental stability and potential in the application of a wide spectrum of technological fields (Gurunathan et al 1999). The immense potential of polyaniline emeraldine salt (PANI) in applications, however, still remains untapped because of its serious drawbacks like insolubility in organic/aqueous solvents, non-processability, modification in structural and electrical properties on storage etc. Considerable degree of research effort is being focussed on overcoming these drawbacks by means of design and synthesis of PANI blends and composites (De Paoli 1997).

The search for newer composite materials with optional properties is an ongoing process and in this line, cyclodextrins deserve a special mention. $\beta$-cyclodextrin (CD) is a well-known sugar oligomer built up from seven glucopyanose units. Each unit is bonded to the other through $\alpha-1,4$-glycosidic linkage and hence the seven units together form a cyclic ring of doughnut or wreath-shaped

*Author for correspondence (smanian2002@yahoo.com) truncated cone. The central portion is a cavity inside of which is lined up with relatively non-polar liphophilic groups. Hence CD is considered as an empty capsule of molecular size and can take up guest molecules of appropriate size to form size-selective host-guest complexes (Szejtli and Osa 1996). This characteristic enables it to function as enzyme-mimic model system. Recently, there is a surge of interest on polymer-cyclodextrin inclusion complexes (Huh et al 2001; Rusa et al 2001) and one study has enlightened the new aspects of inclusion mechanism (Rusa et al 2001). However, there are only a few studies on conducting polymer-CD composite materials. Park et al (2001) and Choi and Park (2000) have investigated the growth and characteristics of nanosized dots and wires of polypyrrole and polyaniline electrochemically synthesized on gold electrodes modified with template molecules such as thiolated $\beta$-cyclodextrin or 4aminothiophenol. Chen et al (2003) reported that sulfonated $\mathrm{CD}$ acts as dopant and also includes polypyrrole chain partially in its cavity which leads to enhanced coplanarity of the chain and improves the conductivity and thermal stability. However, in the case of PANI, Yoshida et al (1999) observed that polyaniline emeraldine base in a solution of CD undergoes a change in conformation from coil to rod at low temperature below $275 \mathrm{~K}$. This occurs because the CD molecules are threaded onto polyaniline chain and confine the conformation to rod- 
like. They further prevent the chemical oxidation i.e. doping of the polymer chain, and ultimately form 'insulated molecular wire'. In a theoretical-cum-experimental study on PANI-CD complexes, Belosludov et al (2002) found that $\mathrm{CD}$ forms $1: 1$ complex with aniline monomer of PANI chain and the guest PANI chain attains a nearplanar configuration which results in a loss of $\pi$-electron delocalization and reduces the conductivity. Besides these, generally studies on conducting polymer-CD complexes are scarce and the supramolecular chemistry of such complexes is still unclear and controversial (Rusa et al 2003). Further, in all the studies CD has been employed in free uncomplexed form. Hence in the present work, a study is performed on the effect of $\mathrm{CD}$ in both the free and inclusion-complexed forms with a guest metal complex, dioxalatodiaquochromate(III).

Dioxalatodiaquochromate(III) (DDC) is an anionic complex of inorganic origin. Application of anionic metal complex as dopant in conducting polyaniline is a new approach. This leads to the synthesis of organic-inorganic hybrid materials with specialized properties. For example, incorporation of $\mathrm{V}_{2} \mathrm{O}_{5}$ (Liracantu and Gomezromero 1999) and $\mathrm{MoS}_{3}$ (Fusalba and Belanger 2000) as composite materials has remarkably improved the electrode properties of conducting polyaniline cathodes. In our previous work (Murugesan and Subramanian 2002), we noted that the octahedral metal oxalate complexes $\left[\mathrm{M}(\mathrm{ox})_{3}\right]$ improve the characteristics of PANI. Moreover, since the complexes are optically active like camphorsulphonic acid, there is a possibility of introduction of optical activity in PANI also.

The present work employs both CD and DDC separately or as their inclusion complex and investigates their effects on the characteristics of PANI in their composite materials. This system combines the complexing property of $\mathrm{CD}$ and the conducting property of PANI and hence the present work is an attempt to study the tuning effect of $\mathrm{CD}$ on the latter.

\section{Experimental}

\subsection{Materials}

Aniline obtained from SD Fine-Chem, India, was purified by distillation over zinc dust before use. $\beta$-cyclodextrin was supplied by Lancaster, England and was used as such. The complex cis-potassium dioxalatodiaquochromate(III) was synthesized in our laboratory by adopting the established procedure (Pass and Sutcliffe 1982) using commercially available analytical grade reagents. Other chemicals e.g. $\mathrm{H}_{2} \mathrm{SO}_{4}, \mathrm{CH}_{3} \mathrm{OH}$ etc were of reagent grade from Qualigens, India and were used as received.

\subsection{Methods}

2.2a Synthesis: PANI was synthesized by chemical polymerization with potassium perdisulphate (PDS) as oxidant (Trivedi 1997). In a typical procedure, $100 \mathrm{ml}$ of $0 \cdot 2 \mathrm{M}$ aniline in aqueous $\mathrm{H}_{2} \mathrm{SO}_{4}$ at $\mathrm{pH} 1$ was polymerized at $0^{\circ} \mathrm{C}$ by adding dropwise $100 \mathrm{ml}$ of precooled $0.2 \mathrm{M}$ PDS with constant stirring. The resulting solution was, therefore, $0 \cdot 1 \mathrm{M}$ with respect to both aniline and PDS. After the addition which continued for more than $1 \mathrm{~h}$, the reaction mixture was kept overnight in a refrigerator for the completion of polymerization. The polymer mass settled was filtered, washed several times with distilled water and ethanol until the washings were colourless and finally with $0 \cdot 1 \mathrm{M} \mathrm{H}_{2} \mathrm{SO}_{4}$ and the sample was dried in an oven at $110^{\circ} \mathrm{C}$ for a few hours.

PANI-DDC and PANI-CD materials were prepared separately by following the same procedure. Appropriate quantities of DDC and CD corresponding to the concentrations of $1.65 \times 10^{-3} \mathrm{M}$ and $1.76 \times 10^{-3} \mathrm{M}$, respectively in the final volume of the polymerization mixture $(200 \mathrm{ml})$ were added initially to the aniline solution in aqueous $\mathrm{H}_{2} \mathrm{SO}_{4}$ and stirred well at $0-5^{\circ} \mathrm{C}$ so that DDCaniline molecular complex and $\mathrm{CD}$-aniline inclusion complex were first formed. In the case of PANI-CD + DDC material synthesis, first the $\mathrm{CD}+\mathrm{DDC}$ inclusion complex of $1: 1$ molar ratio was formed in aqueous $\mathrm{H}_{2} \mathrm{SO}_{4}$ by dissolving the required quantities of both the samples and stirring the solution well for a few hours at $0-5^{\circ} \mathrm{C}$. Then aniline was added to the same solution, stirred well and cooled. After ensuring complex formation in each case by UV-vis spectra, aniline was polymerized with PDS in the same way. In all these cases, after completion of polymerization, the mother liquor was collected carefully and preserved for chemical analyses. Then the polymer sample was subjected to washing and drying as described above.

2.2b Chemical analyses: The mother liquor contained not only unincorporated DDC and CD but also impurities from aniline polymerization like benzoquinone, hydroquinone, oligomer etc. They could provide interference in the estimation of DDC and CD and hence mother liquor from aniline polymerization in aqueous $\mathrm{H}_{2} \mathrm{SO}_{4}$ alone, performed in identical condition was considered as blank and subjected to chemical treatment in the same way as the other mother liquors.

In the estimation of DDC, mother liquor was first treated with sufficient quantity of aqueous $\mathrm{NH}_{3}$ to make it alkaline and then agitated for $4 \mathrm{~h}$ in a shaker. In this process the metal complex dissociated into free metal ion and oxalate anion and the latter was estimated spectrophotometrically (Pass and Sutcliffe 1982) with acidified $\mathrm{KMnO}_{4}$ solution by measuring the absorbances at $\lambda=$ $545 \mathrm{~nm}$. A calibration graph obtained with a series of standard oxalate solutions was used in the estimation of unknown oxalate.

$\mathrm{CD}$ in the mother liquor was estimated by anthrone reagent method (Scott and Melvin 1953) which is also basically a spectrophotometric method. In this, $5 \mathrm{ml}$ of 
$0 \cdot 18 \%(\mathrm{w} / \mathrm{v})$ anthrone reagent in concentrated $\mathrm{H}_{2} \mathrm{SO}_{4}$ was taken in a test tube. To this, diluted mother liquor solution of volume $3 \mathrm{ml}$ was pipetted out and the two-layer solution was mixed vigorously in a cold water bath shaker $\left(\sim 25^{\circ} \mathrm{C}\right)$ without allowing the solution to raise its temperature. After thorough mixing, the solution was heated to $90^{\circ} \mathrm{C}$ in a hot water bath and maintained at the same temperature for $7 \mathrm{~min}$ during which a green colour was developed in the solution. After cooling to room temperature with cold water bath, the absorbance of the solution at $\lambda=625 \mathrm{~nm}$ was measured. For calibration purpose, a series of standard CD solutions in the concentration range $0 \cdot 35-2 \cdot 11 \times 10^{-5} \mathrm{M}$ was used.

After making correction for dilution factor and using blank from PANI mother liquor, the unincorporated DDC and CD were estimated. Estimation was made in duplicate and the values obtained were close but the average of the two was considered for final estimation.

The sulphate and moisture contents in the polymer materials were determined as described in our previous paper (Murugesan and Subramanian 2002).

2.2c Spectral characterization: The UV-vis absorption spectra of the complex DDC, inclusion complex CD + DDC alone or with aniline in $1.0 \mathrm{M} \mathrm{H}_{2} \mathrm{SO}_{4}$ and of PANI in $1.0 \mathrm{M} \mathrm{H}_{2} \mathrm{SO}_{4}$ were recorded with a Perkin Elmer UVvis spectrophotometer (Lambda 3B model) in matched $1 \mathrm{~cm}$ quartz cuvettes. FTIR spectra of all PANI materials in $\mathrm{KBr}$ pellet were obtained from JASCO FTIR $410 \mathrm{spec}-$ trophotometer.

2.2d Conductivity measurement: Electrical conductivity of the materials was measured by a standard collinear four-probe technique. Pellets were made from the materials with a hydraulic press (KIMAYA Engineers, India, model WT-324) by applying 3 metric ton pressure and they had the dimensions of diameter, $13 \mathrm{~mm}$ and thickness, $\sim 2 \mathrm{~mm}$. $I-V$ measurements were made by making a gentle contact at different positions of the pellet with a gold-tipped collinear four-probe set-up (inter probe spacing $=2 \mathrm{~mm}$ ) as described in literature (Wieder 1979) and drawing current from a constant power supply unit (Scientific Equipment and Services, Roorkee, Model DFP02). From the slopes of the $I-V$ plots which were linear and overlapping for different position measurements, the resistances were determined and using the dimension the conductivities were calculated. Determination of conductivity by this instrumental set-up was simple and quick and the values were closely agreeable, within experimental error, with the conductivity values determined by the standard square four-probe technique involving silver paste ohmic contacts (Wieder 1979).

For the same pellets, conductivities were also measured after exposing them to saturated methanol vapour for about $30 \mathrm{~min}$ in a closed chamber. The experiment was repeated two or three times after reexposing the pellets each time to air for regeneration and conductivities were measured as before. The values obtained were completely reproducible.

\section{Results and discussion}

\subsection{Chemical analyses and stoichiometry of DDC and CD}

The chemical analysis for DDC in mother liquors of PANI-DDC and PANI-CD + DDC shows that there is no measurable DDC and that almost all the DDC initially taken has been incorporated into the materials. In our previous study also (Murugesan and Subramanian 2002), it was established that all the stable metal oxalates were incorporated into PANI without any loss. This is quite possible because, as is evident from the following discussion, DDC forms a strong molecular complex with protonated aniline and aniline has about 60 times higher concentration than DDC, leaving no room for free/undoped DDC.

Anthrone method yielded the result that $97.5 \%$ of CD has been incorporated into PANI. Taking into account the sulphate content (1-1.12 unit per aniline monomer) and the yield of polymerized aniline, the stoichiometry of aniline : DDC and aniline : CD are found to be 15.13:1 and $15 \cdot 74: 1$, respectively. It can be stated that roughly for every 15 aniline monomer units in the PANI chain, there is one DDC in PANI-DDC and one CD in PANI$\mathrm{CD}$ and PANI-CD + DDC materials.

\subsection{Electronic spectral characterization}

The chromium complex DDC, and its inclusion complex with $\beta-C D(C D+D D C)$ were characterized by their electronic spectra. Figure 1 shows the electronic spectra in visible region. The complex DDC exhibits four absorption bands at 804, 561, 414 and $340 \mathrm{~nm}$ (spectrum 1). Since the complex contains two different types of ligands, oxalato and aquo, it is not octahedral and can, therefore, undergo Jahn-Teller distortion (Huheey et al 1993) leading to the split of three higher energy electronic states into six. Theoretically, thus, six electronic absorption bands (four in the visible region and two in the UV region) are possible (Huheey et al 1993). The observation of four absorption bands (for DDC complex) in the visible region confirms firmly its formation with expected structure in the cis-configuration. Spectrum 2 in figure 1 depicts the electronic spectrum of the same DDC in its host-guest inclusion complex with CD. As evident from the spectrum, there is no change in the peak position except a slight increase in absorption for the first two bands and a considerable decrease for the fourth band (beginning from higher wavelength) is noticeable. The first two bands are metal-centred ( $d-d$ transitions) while the fourth one involves ligand to metal charge transfer (LMCT) transition 
(Huheey et al 1993). Considerable decrease in absorption of fourth band, hence, suggests the reduced interaction between oxalato ligand and $\mathrm{Cr}^{3+}$ ion at the orbital level, owing to the interaction between $\mathrm{CD}$ and DDC in the complexed state. Thus the inclusion complex formation between CD and DDC is confirmed by electronic spectroscopy.

Both DDC and the inclusion complex CD + DDC carry one negative charge and hence they can act as dopants for PANI which could be discussed by their electronic spectroscopy in the presence of aniline. In $1.0 \mathrm{M} \mathrm{H}_{2} \mathrm{SO}_{4}$ medium, aniline exists in the protonated form with $-{ }^{\oplus} \mathrm{NH}_{3}$ moiety which is analogous to PANI containing protonated imine centre, $\mathrm{C}={ }^{\oplus} \mathrm{NH}$. Hence, aniline in $\mathrm{H}_{2} \mathrm{SO}_{4}$ was employed to observe the spectral changes of DDC and CD + DDC (spectra 3 and 4 in figure 1, respectively). Conspicuously, there is a drastic change in their absorption spectra with protonated aniline. In the case of DDC, there is an enormous increase in absorption together with a blueshift in first and third bands, while the fourth band, because of blueshift obscures into the UV region and could not be observed. With CD + DDC inclusion complex, the same spectral course with a marginal decrease in absorbance is observed. This drastic change in spectra

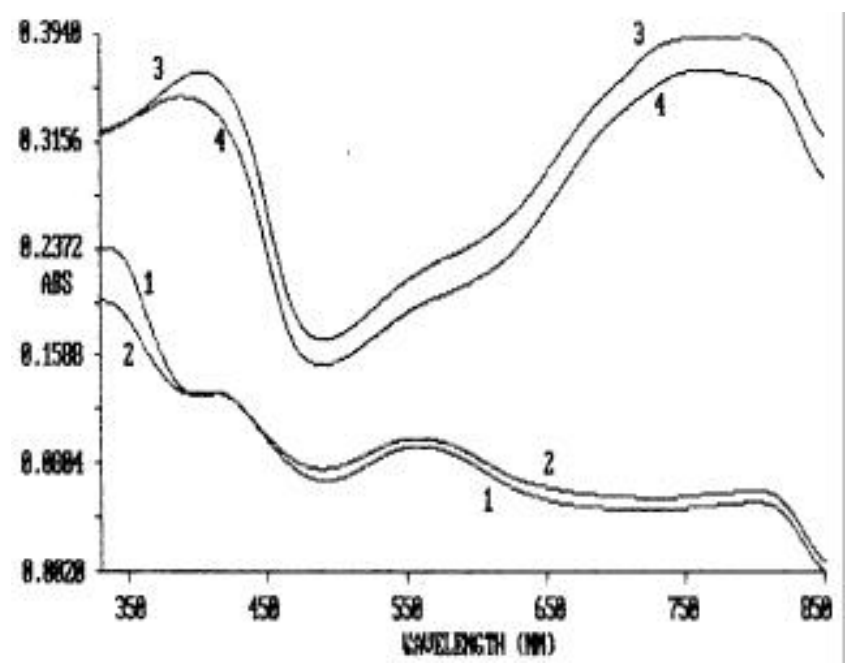

Figure 1. Electronic absorption spectra in $1.0 \mathrm{M} \mathrm{H}_{2} \mathrm{SO}_{4}$ of (1) DDC, (2) CD + DDC, (3) DDC + aniline and (4) CD + DDC + aniline. demonstrates a strong interaction between protonated aniline and DDC/CD + DDC complex species. It is likely that an ion-pair like interaction involving coulombic forces, operates between the protonated and positively charged aniline and the anionic DDC/CD + DDC complex species. Extending this observation to PANI, the two complexes, DDC and CD + DDC, could act as dopants for PANI.

\subsection{Electrical conductivities}

The electrical conductivities of the four materials, PANI, PANI-CD, PANI-DDC and PANI-CD + DDC are given in table 1. Incorporation of $\mathrm{CD}$ and $\mathrm{DDC}$ reduces the conductivity of PANI by $58 \%$ and $49 \%$, respectively while the CD + DDC host-guest complex enhances it by $27 \%$. These contrasting influences originate from the characteristically differing interactions of $\mathrm{CD}, \mathrm{DDC}$ and $\mathrm{CD}+$ DDC inclusion complex with PANI which could be understood from the spectral characterizations.

\subsection{IR spectral characterization of PANI materials}

Figure 2 illustrates FTIR spectra of all the four materials. The characteristic stretching bands for quinoid, benzenoid, imine and secondary amine $\mathrm{N}$ centres and aromatic $\mathrm{C}-\mathrm{H}$ in-plane and out-plane bending vibrations occur at $1563,1477,1301,1224,1125$ and $802 \mathrm{~cm}^{-1}$, respectively (Boyer et al 1998) for PANI (spectrum 1). These vibrational bands are modified in peak position and relative intensity on incorporation of CD, DDC or their inclusion complex into PANI. Of the various bands mentioned, the prominent ones pertaining to conductivity are the benzenoid, amine and $\mathrm{C}-\mathrm{H}$ in-plane bands.

$\mathrm{CD}$ and DDC incorporations redshift the benzenoid and $\mathrm{C}-\mathrm{H}$ in-plane bands and blueshift the amine band. Moreover, the intensity and bandwidth of $\mathrm{C}-\mathrm{H}$ in-plane band relative to others are reduced. Hence, it is inferred that they affect the chain stretching and $\pi$-electron delocalization of PANI chain and cause a decrease in conductivity. A recent study on the inclusion complex of PANI with CD has proposed a decrease in conductivity (Belosludov et al 2002). In our previous study (Murugesan and Subramanian 2002), we observed that trioxalatochromate(III)

Table 1. Conductivity of PANI materials.

\begin{tabular}{|c|c|c|c|c|}
\hline \multirow[b]{2}{*}{ Material } & \multicolumn{2}{|c|}{ Conductivity $\left(\sigma\left(\mathrm{S} \mathrm{cm}^{-1}\right)\right)$} & \multirow{2}{*}{$\begin{array}{l}\text { Normalized } \\
\text { conductivity }\end{array}$} & \multirow[b]{2}{*}{$\%$ change in $\sigma$} \\
\hline & Native & Methanol exposed & & \\
\hline PANI & $2 \cdot 94$ & $2 \cdot 74$ & $0 \cdot 068$ & $6 \cdot 80$ \\
\hline PANI-CD & 1.71 & $1 \cdot 18$ & $0 \cdot 309$ & $30 \cdot 90$ \\
\hline PANI-DDC & $1 \cdot 45$ & - & - & - \\
\hline PANI-CD + DDC & 3.74 & $4 \cdot 19$ & $-0 \cdot 120$ & $-12 \cdot 03$ \\
\hline
\end{tabular}


(analogous to DDC in the present work) complex-doped PANI has a lower conductivity than PANI. These two literature reports supplement the observation of the present work. The decrease in conductivity by CD and DDC has correlation with the extent of amine band shift effected by these compounds. DDC with greater shift from $1224 \mathrm{~cm}^{-1}$ of PANI to $1256 \mathrm{~cm}^{-1}$ of PANI-DDC causes a larger decrease in conductivity while $\mathrm{CD}$ exhibits a lesser shift (1224 to $1243 \mathrm{~cm}^{-1}$ ) and a corresponding smaller decrease in conductivity.

With the inclusion complex CD + DDC, however, no such perceptible band shift is observed and the spectrum looks like that of PANI. Yet some differing features are noticeable. The $\mathrm{C}-\mathrm{H}$ in-plane bandwidth and the intensity of amine band relative to imine band are increased. These features are, no doubt, suggestive of improved electron delocalization in PANI-CD + DDC material than in PANI and hence substantiate the higher conductivity in the former. A similar observation in IR features and increase in conductivity has been made on leucoemeraldine polyaniline with concurrent alkylation and doping by alkyl halides (Zhao et al 2000).

\subsection{Visible spectral characterization of PANI materials}

The visible absorption spectra of PANI materials in $1.0 \mathrm{M} \mathrm{H}_{2} \mathrm{SO}_{4}$ are displayed in figure 3. Three spectral transitions at 770,430 and $358 \mathrm{~nm}$ are observed which agree well with many literature reports (Sindhimeshram and Gupta 1995; Rao et al 2000). The first one appears as a broad band, the second one a shoulder and the third one a hump and are assignable to the presence of emeraldine

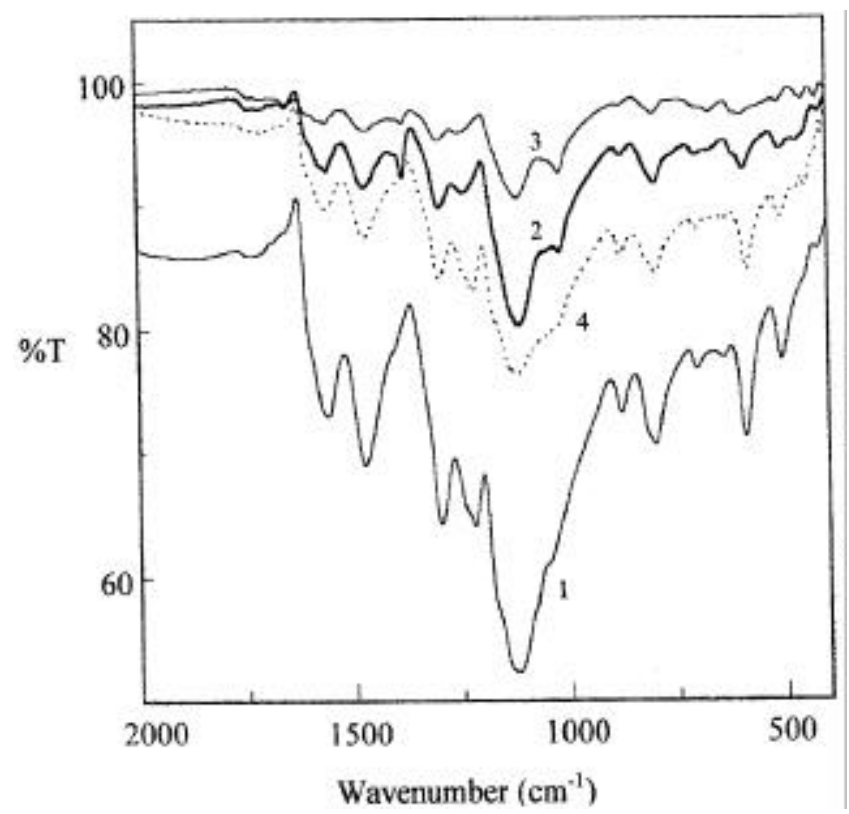

Figure 2. FTIR spectra of polyaniline materials: (1) PANI, (2) PANI-CD, (3) PANI-DDC and (4) PANI-CD + DDC. salt, the polaron and the HOMO-LUMO $\pi-\pi^{*}$ transition, respectively. Though the general shape of the spectra of all the four materials appears to be identical, one feature, viz. the difference in $\lambda_{\max }$ of the higher wavelength emeraldine salt band, is consistent with the IR feature. For PANI and PANI-CD + DDC materials having higher conductivity, this band occurs at $777 \mathrm{~nm}$ but for PANI$\mathrm{CD}$ and PANI-DDC, it occurs at slightly lower-wavelengths i.e. 767 and $765 \mathrm{~nm}$, respectively. A higher wavelength specifies a lower band gap and hence a larger conductivity. Further, the absorbance of the materials at the emeraldine salt band is in the increasing order.

PANI $<$ PANI-CD $<$ PANI-DDC $<$ PANI-CD + DDC.

When analysed on the basis of the experimental fact that the aliquots for these spectra were prepared from the same weight of polymer sample in the same volume of solvent, this order indicates the order of solubility of these materials in $1.0 \mathrm{M} \mathrm{H}_{2} \mathrm{SO}_{4}$.

\subsection{Molecular dispositions and interactions}

Results so far discussed in the present study clearly point out that CD and DDC play declining roles in conductivity of PANI. Their inclusion complex, however, remains so favourable that their composite material with PANI could emerge as a better conductor and a more soluble material in $1.0 \mathrm{M} \mathrm{H}_{2} \mathrm{SO}_{4}$. This emergence is possible only when the molecular disposition and mode of interaction of CD + DDC are different from those of individual CD and DDC entities in PANI-association. Belosludov et al (2002) in their study on PANI-CD inclusion complex have found that PANI attains a rod-like conformation in CD cavity such that the two adjacent benzene rings have near-planar arrangement which reduces the extent of $\pi$-orbital over-

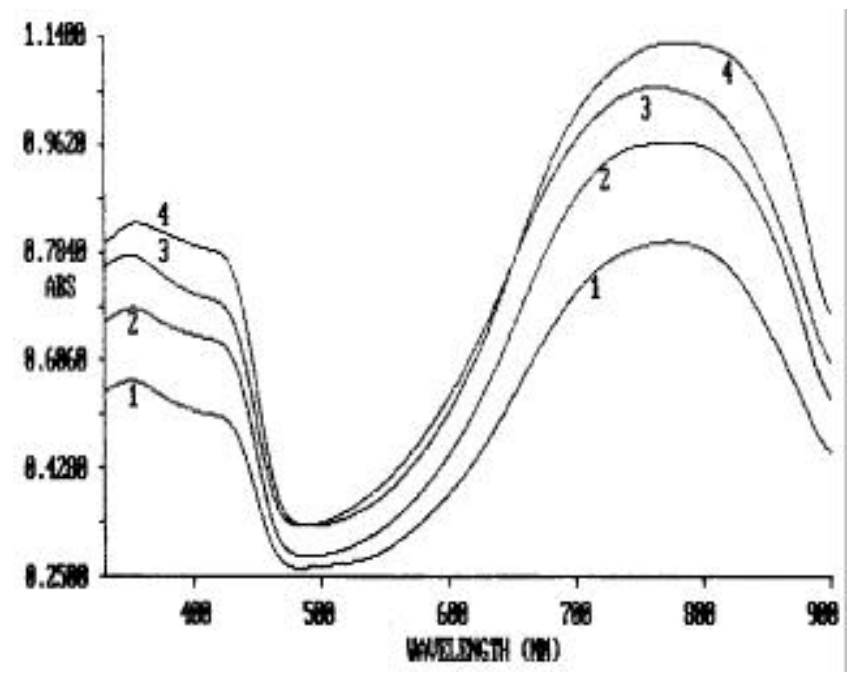

Figure 3. UV-vis absorption spectra in $1.0 \mathrm{M} \mathrm{H}_{2} \mathrm{SO}_{4}$ of (1) PANI, (2) PANI-CD, (3) PANI-DDC and (4) PANI-CD + DDC. 
lap, breaks up the electron channels and decreases the conductivity. The present work also concludes with a decrease in $\pi$-electron delocalization and the resulting decrease in conductivity for PANI-CD material. But in the present experimental conditions where aniline and $\mathrm{CD}$ are in $15: 1$ molar ratio, all the PANI molecules and/ or the entire PANI chain may not be in inclusioncomplexed form with CD. Nevertheless, few CD molecules encapsulating PANI chain, as evident from IR spectra, involve in hydrogen bonding with imine-amine $\mathrm{N}$ centres, facilitate the transfer/polarization of electron cloud from chain segment to themselves and thereby disturb the $\pi$-electron delocalization (see decrease in wavenumbers of vibrational bands). DDC also interacts similarly but, of course, lying adjacent to the chain. However, for $\mathrm{CD}+\mathrm{DDC}$ inclusion complex, the interaction must be different. CD is reported (Szejtli 1998) to have the dimensions: internal cavity diameter, $7 \cdot 8 \AA$ A outer diameter, $15 \cdot 3 \AA$ and cylindrical depth, 7.8 $\AA$. In our previous report (Murugesan and Subramanian 2003), we presented that the distance between two stacks in the 2D stacking arrangement of PANI chains is $16.3 \AA$. It is interesting to note that the inter-stack distance in PANI is matchable with $\mathrm{CD}$ outer diameter. Hence, in the material PANI$\mathrm{CD}+\mathrm{DDC}$, the inclusion complex $\mathrm{CD}+\mathrm{DDC}$ together with any interacting water (solvent) molecules could seat in between two stacks in such a way that the negative end of encapsulated DDC interacts with imine-amine $\mathrm{N}$ centres. In this arrangement, the hydrogen bonding interactions of $\mathrm{CD}$ and DDC get saturated within themselves and/or in interactions with the held-up water molecules so that it is not extended to the adjoining PANI chain. Such an arrangement would not only stretch the PANI chain but also prevent any coiling effect, which ultimately leads to enhanced $\pi$-electron delocalization and conductivity. Thus in PANI-CD material, CD encapsulates PANI chain randomly and performs its role but in PANI-CD + DDC, the CD + DDC inclusion complex lies in between the PANI chains and acts as a dopant. In Ppy$\mathrm{CDSO}_{3}^{-}$composite material, Chen et al (2003) have shown that about $20 \%$ of $\mathrm{CDSO}_{3}^{-}$acts as dopant lying in between Ppy chains and the remaining $80 \%$ involves in encapsulation.

\subsection{Interaction with methanol vapour}

In order to get further insight into the molecular disposition and mode of interaction just described for PANI-CD and PANI-CD + DDC, a further study on interaction behaviours of these materials with a third molecular component, viz. methanol, was performed. Table 1 presents the change in conductivity together with normalized conductivity and the percentage change in conductivity (pcc) of these materials after exposure to methanol vapour for $30 \mathrm{~min}$ in a closed chamber. As expected, PANI, PANI$\mathrm{CD}$ and PANI-CD + DDC show contrasting behaviours, the former two a decrease in conductivity and the latter an increase. In our previous work (Anitha and Subramanian 2003), we reported a pcc of $6 \cdot 3$ for PANI which closely agrees with the present value. The opposite trends in normalized conductivity clearly reveal that methanol associates with PANI and PANI-CD in a similar way but differently with PANI-CD + DDC. Difference IR spectra (spectrum of the methanol exposed material - native material) shown in figure 4 representatively for PANI-CD and PANI-CD + DDC provide clues to the varying methanol association. The absorbance sign difference of the two spectra indicates that methanol enhances the overall absorbance of PANI-CD but reduces it for PANI-CD + DDC. Further, the vibrational bands of quinoid and amine groups appear blueshifted in PANI-CD but redshifted in PANI-CD + DDC. For C-H in-plane deformation band, the shift is just in the reverse directions. For PANI-CD (and hence for PANI also, because of similar effect) a possible molecular arrangement of methanol association is that it occupies an interchain position and forms hydrogen bond with amine group through $\mathrm{O}$ donation. This is similar to the arrangement reported by Tan and Blackwood (2000). Obviously, methanol injects electrons into PANI-CD and nullifies polarons and bipolarons leading to a decrease in conductivity. In PANI-CD + DDC, since $\mathrm{CD}+\mathrm{DDC}$ already interacts with imine-amine centres as dopant seating in between the chains, methanol finds no place there. In a likely arrangement, it could first coordinate with the $\mathrm{CD}+\mathrm{DDC}$ inclusion complex through hydrogen bonding, then lie parallel to benzene ring in flat configuration and exhibit dispersion forces, drawing the electron cloud out of benzene ring via orbital overlap.

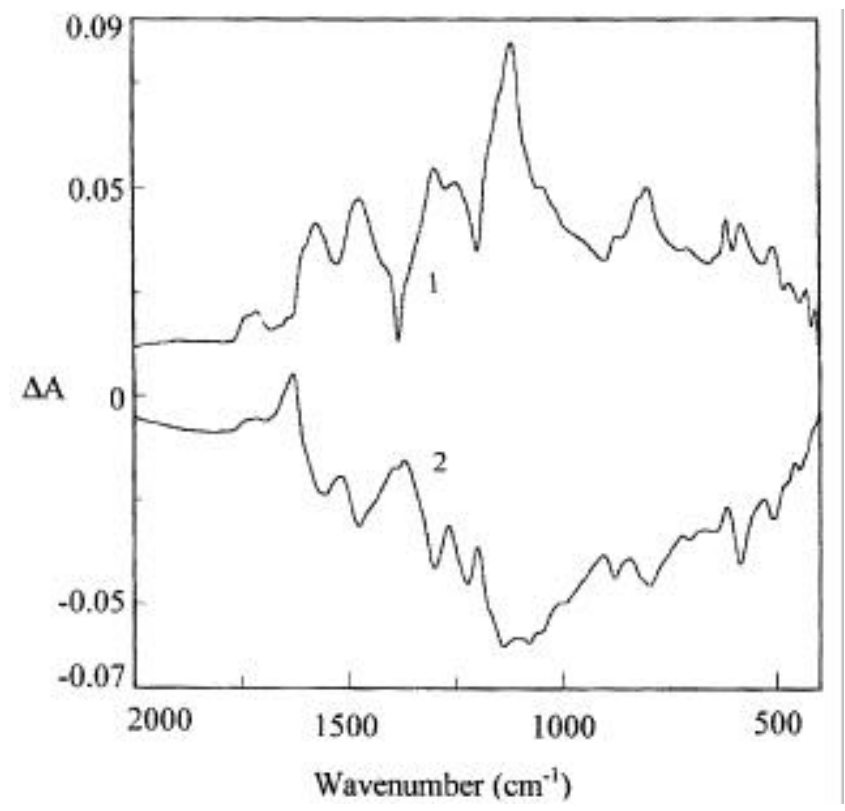

Figure 4. FTIR difference absorption spectra ( $\Delta A=A_{\text {exposed }}-$ $\left.A_{\text {native }}\right)$ of polyaniline materials after methanol vapour exposure: (1) PANI-CD and (2) PANI-CD + DDC. 
This extended electron delocalization may be responsible for the observed increase in conductivity. Thus the methanol effect on conductivity provides an indirect evidence for the varying molecular dispositions of PANI$\mathrm{CD}$ and PANI-CD + DDC composite materials.

\section{Conclusions}

Conductivity and spectral studies of the present work reveal that the sugar oligomer $\mathrm{CD}$ and the anionic complex DDC reduce the conductivity of PANI by about half. Yet their inclusion complex enhances it. All the three, on the contrary, appear to enhance the solubility of PANI in $1.0 \mathrm{M} \mathrm{H}_{2} \mathrm{SO}_{4}$. In PANI-CD material, $\mathrm{CD}$ encapsulates the PANI chain randomly and reduces the conductivity through hydrogen bonding interaction. Contrarily, in PANI$\mathrm{CD}+\mathrm{DDC}$ material, CD + DDC inclusion complex acts as a dopant, seating in between the PANI chains. This disposition seemingly improves the extended conformation and $\pi$-electron delocalization of PANI chain and hence its conductivity. Interaction with methanol vapour pinpoints the distinct role of $\mathrm{CD}+\mathrm{DDC}$ in comparison with $\mathrm{CD}$ in altering the electrical property of PANI.

\section{Acknowledgement}

The financial assistance offered by UGC, New Delhi, is gratefully acknowledged.

\section{References}

Anitha G and Subramanian E 2003 Sens. Actuators B Chem. 92 49

Belosludov R V, Mizuseki H, Ichinoseki K and Kawazoe Y 2002 Jpn. J. Appl. Phys. 412739

Boyer M I, Quillard S, Rebourt E, Louarn G, Bulsson J P, Monkman A and Lefrant S 1998 J. Phys. Chem. B102 7382

Chen W, Wan X, Xu N and Xue G 2003 Macromolecules 36 776

Choi S J and Park S M 2000 Adv. Mater. 121547
De Paoli M A 1997 Conductive polymer blends and composites, in Conductive polymers: Synthesis and electrical properties, Handbook of organic conductive molecules and polymers (ed.) H S Nalwa (Chichester: John Wiley and Sons Ltd) Vol. 2, p. 773

Fusalba F and Belanger D 2000 Electrochim. Acta 453877

Gurunathan K, Murugan A V, Marimuthu R, Mulik U P and Amalnerkar D P 1999 Mater. Chem. Phys. 61173

Huh K M, Ooya T, Sasaki S and Yui N 2001 Macromolecules 342402

Huheey J E, Keiter E A and Keiter R L 1993 Inorganic chemistry (New York: Harper Collins College Publishers) 4th ed. p. 448

Liracantu M and Gomezromero P 1999 Int. J. Inorg. Mater. 1111

Murugesan R and Subramanian E 2002 Bull. Mater. Sci. 25613

Murugesan R and Subramanian E 2003 Mater. Chem. Phys. 80 731

Park S M, Lee J Y and Choi S J 2001 Synth. Met. 1211297

Pass G and Sutcliffe H 1982 Practical inorganic chemistry (New York: Chapman and Hall Ltd) 2nd ed. pp 94 \& 182

Rao P S, Anand J, Palaniappan S and Sathyanarayana D N 2000 Eur. Polym. J. 36915

Rusa C C, Luca C and Tonelli A E 2001 Macromolecules 34 1318

Rusa C C, Fox J and Tonelli A E 2003 Macromolecules 36 2742

Scott T A and Melvin E R 1953 Anal. Chem. 251656

Sindhimeshram D C and Gupta M C 1995 Indian J. Chem. A34 260

Szejtli J 1998 Chem.Rev. 981743

Szejtli J and Osa T 1996 Comprehensive supramolecular chemistry, cyclodextrins (Kidington: Pergamon, Elsevier Science Ltd) ch. 2 and 5, Vol. 3

Tan C K and Blackwood D J 2000 Sens. Actuators B Chem. 71 184

Trivedi D C 1997 Polyanilines, in Conductive molecules and electric properties, Handbook of organic conductive molecules and polymers (ed.) H S Nalwa (Chichester: John Wiley and Sons Ltd) Vol. 2 p. 505

Wieder H H 1979 Laboratory notes on electrical and galvanomagnetic measurements (Amsterdam: Elsevier Scientific Publishing Company) ch. 1, pp 11 and 17

Yoshida K, Shimomura T, Ito K and Hayakawa R 1999 Langmuir 15910

Zhao B, Neoh K G and Kang E T 2000 Chem. Mater. 121800 